\section{Diagnostic Yield of Sural Nerve Biopsy: Study from a Tertiary Care Referral Centre in India}

\section{Abstract}

Background: Peripheral neuropathies are a heterogeneous group of disorders with varying etiologies. A systematic approach is required for patient evaluation for cost effective diagnosis.

Aims: Present study conducted at a tertiary care referral centre aimed to evaluate the clinical profile and usefulness of sural nerve biopsy.

Material and method: The study was conducted on 75 patients attending the neurology outpatient department/those admitted in the wards. All patients were subjected to a detailed clinical, neurological and necessary biochemical investigations. Those patients with an inconclusive result were subjected to a sural nerve biopsy with the results being interpreted as diagnostic, contributory or noncontributory.

Results: Out of the 75 patients enrolled 36 underwent nerve biopsy. Amongst those 18 turned out to have Hansen's disease, 8 vasculiis, 2 CIDP, 1 diabetes and 7 patients remained undiagnosed. Overall in 15 cases the biopsy was diagnostic, in 14 cases it was contributory, while in 7 cases it was noncontributory. Nerve biopsy proved more beneficial in patients with a multiple mononeuropathy pattern $(p<0.003)$. Overall in about $80 \%$ of patients nerve biopsy proved to be worthwhile

Conclusion: Nerve biopsy has a good diagnostic yield if done in properly selected cases. This is especially so for the asymmetrical neuropathies particularly infectious causes like leprosy and vasculitis.

Keywords: Nerve biopsy; Peripheral neuropathy; Sural nerve

\section{SP Patidar, Deepika Joshi, Mishra VN, Chaurasia RN, Ansari AZ and Niraj Kumar}

\author{
Department of Neurology, Institute of \\ Medical Sciences, Banaras Hindu University, \\ Varanasi, India
}

\section{*Corresponding author: Deepika Joshi \\ ¡ drdeepikajoshi73@gmail.com}

Department of Neurology, Institute of Medical Sciences, Banaras Hindu University, Varanasi, India, 221005.

Citation: Patidar SP, Joshi D, Mishra VN, et al. Diagnostic Yield of Sural Nerve Biopsy: Study from a Tertiary Care Referral Centre in India. J Neurol Neurosci. 2016, 6:1.

Received: May 06, 2015; Accepted: June 15, 2015; Published: June 18, 2015

\section{Introduction}

Peripheral neuropathies are amongst the most common disorders in patients attending neuromuscular clinic. A step wise systematic approach comprising a good clinical history, a thorough neurological and systemic examination, electro diagnostic studies and relevant biochemical tests should be undertaken. Nerve biopsy should only be performed when there is suspicion of certain disorders, particularly amyloidosis, vasculitis, leprosy, and tumor infiltration. Nerve biopsy is rarely necessary for the diagnosis of CIDP and should primarily be used to exclude other etiologies. The yield of nerve biopsy depends on a number of factors, including selection of patients, expertise of the laboratory, and techniques used. A recent prospective study has shown that nerve biopsy improves treatment in an estimated $60 \%$ of patients [1], a figure close to that of an earlier retrospective study [2].
With hereditary neuropathies, it is now seldom necessary to perform a morphological study of a nerve biopsy specimen. In multifocal neuropathy, nerve biopsy more often contributes to the diagnosis than in the other patterns of neuropathy.

Although sural nerve biopsy has been considered a standard method of diagnosing vasculitic neuropathy, the procedure yields unequivocal evidence of vasculitis in only $20 \%$ of patients in whom biopsies are performed for this indication $[3,4]$.

A recent multicenter prospective study confirmed the higher yield for performing a biopsy of the superficial peroneal nerve combined with a peroneus brevis muscle biopsy to search for vasculitis because of the higher frequency of involvement of the peroneal nerve in vasculitic neuropathy and the frequent involvement of muscle arteries [5]. 


\section{Patients and Method}

The present study was carried out at a tertiary care 1000 bedded referral hospital in eastern part of India, over a period of 18 months from April 2009 to September 2010.

This study was conducted on Seventy Five patients suffering from peripheral neuropathy. These were referred to neurology department for evaluation and included in the study after proper written consent. The neuropathy was considered disabling when sensorimotor deficit of peripheral origin leads to impairment of activity of daily living. Patients suffering from a disabling neuropathy of known origin were included in the study only when uncommon manifestations were present. Neurological interview and examination was carried out in a standardized manner. A detailed evaluation was performed on all individuals to record the number and distribution of affected nerves. Nerve thickening was ascertained by comparing one nerve with its counterpart on the contra lateral side. Sensory impairment, motor deficit, and disability/deformity status were assessed by using standard methods. Individual muscle power was graded according to the method described by the Medical Research Council (MRC) of London [6].

The distribution of motor and sensory symptoms were recorded as distal symmetrical, distal asymmetrical, or focal or multifocal. A family history of neurological disease, the concurrence of other diseases or possible intoxication (medication, alcohol or other toxic agents) were also recorded.

All patients had routine blood tests, biochemical assays and protein electrophoresis. Other tests were performed only when required in the clinical context.

Routine electrophysiological tests were carried out in all patients comprising motor and sensory nerve conduction and $\mathrm{F}$ waves. The median and ulnar nerves in the upper limbs and the Tibial, Common Peroneal and Sural nerves were evaluated in the upper limbs. In cases of symmetrical neuropathies one upper limb and one lower limb were evaluated while in asymmetrical neuropathies at least three limbs were evaluated. The following variables were measured: distal motor latency, motor and sensory conduction velocity, the amplitude of the compound muscle action potential, $\mathrm{F}$ wave and sensory nerve action potential. The variables were considered abnormal when they exceeded the limits of normality by 2 SDs. Normal data were prepared for our laboratory. When required in the clinical context, muscles were examined using concentric needles.

Those patients in whom the clinical, electro diagnostic, and the biochemical tests were inconclusive were subjected to a sural nerve biopsy.

Sural nerve biopsy was carried out under aseptic precautions after taking a written informed consent. The procedure was done under local anesthesia using $1 \%$ lignocaine which was infiltrated behind lateral malleolus. Incision was given along the short saphenous vein. The scarpa's fascia was divided with blunt dissection exposing the nerve and vein which was separated. Nerve was ligated at the proximal end and was cut distal to ligature. A $2.5 \mathrm{~cm}$ length of nerve was taken out.
Gluteraldeyde/formalin fixed nerve biopsies were processed for paraffin embedding and sectioned in transverse and longitudinal planes. Four- 6 micron thick sections were routinely stained with hematoxylin-eoisn (HE) for morphological examination and masson's trichrome (MAT) for collagen. For the Kultshitsky-pal (K-pal) stain for myelin, a small segment of fixed nerve was placed overnight in Fleming's solution and processed the next day for paraffin embedding. Final diagnosis was made after biopsy. If diagnosis was not evident then cryptogenic neuropathy was labeled.

To evaluate the yield of nerve biopsies, we referred to the criteria published by Midroni et al. and Argov et al . Diagnostic biopsies showed abnormalities specific or highly suggestive of a definitive diagnosis. Contributory biopsies provided information that was either essential or helpful for the patient's management. Noncontributory biopsies did not influence patient's management and was not revealed any significant information helping in diagnosis or management of patient. All data entered in SPSS 16.0 version and analyzed. Significant difference between proportions is tested by Fishers's exact probability test.

\section{Results}

Out of total 75 patients, 36 patients selected for nerve biopsy. Thirty nine (52\%) patients were diagnosed with detailed clinical electrophysiological and routine investigations. The mean age in biopsy group patients was $43.19 \pm 22.62$ years, range from 8 to 72 years. Patients were dispersed in all age groups with two peaks between $11-30$ and $61-80$ years age group $36 \%$ in each. Male: female were 2.2:1. Total duration of illness before biopsy varied from 1 month to 20 years, median was 12 months. Multiple mononeuropathy was present in $67 \%$ and polyneuropathy was found in $33 \%$. Sensorimotor (72.22\%) type of neuropathy was most common clinical and electrophysiological diagnosis while predominant sensory presentation was seen in $27.8 \%$ of patients. Sensory symptoms were symmetrical in 14 and asymmetrical in 25 patients. Weakness was present in 34 (94\%) of patients, which was symmetrical in $44 \%$ and asymmetrical in $56 \%$ patients. Other results are depicted in Table 1 . Final diagnosis could be made in $80 \%$ of patients. Diagnosis was Hansen's disease in $50 \%$, Vasculitis in $22 \%$, CIDP in $5.5 \%$ and diabetes in $2.78 \%$ (Table 2).

In fifteen cases the nerve biopsy had changed the preferred diagnosis or gave diagnosis otherwise even not suspected. This group was called as 'Diagnostic'. In 14 cases the biopsy had contributed by confirming a diagnosis which had already been suspected. This group was called as 'Contributory'.

In seven cases the biopsy did not contribute to the diagnosis and thus those patients remained undiagnosed and were called as 'Idiopathic' with the nerve biopsy being 'Noncontributory'. Out of these 'Idiopathic' cases, one case was suspected cryoglobulinemic vasculitic neuropathy secondary to Hepatitis $C$ infection. Another patient suspected to have B12 deficiency neuropathy, remained unproved after investigation. This patient responded well to parenteral B12 treatment during 6 month follow up.

Out of eighteen leprosy patients nerve biopsy revealed epitheloid granuloma in $39 \%$, AFB positive in $28 \%$ and inflammatory infiltrate 
Table 1 Clinical and electrophysiological profile.

\begin{tabular}{|c|c|c|c|c|}
\hline & Diagnostic & Contributory & Noncontributory & Total (\%) \\
\hline No. of patients & 15 & 14 & 7 & 36 \\
\hline \multicolumn{5}{|l|}{ Clinical presentation } \\
\hline Multiple Mononeuropathy & 11 & 12 & 1 & $24(67)$ \\
\hline Polyneuropathy & 4 & 2 & 6 & $12(33)$ \\
\hline Impaired JP/vibration & 10 & 8 & 3 & 21 \\
\hline Diminished DTRs & 12 & 5 & 2 & 19 \\
\hline Tingling & 14 & 11 & 3 & 28 \\
\hline Numbness & 12 & 13 & 4 & 29 \\
\hline \multicolumn{5}{|l|}{ Sensory Motor involvement } \\
\hline Sensory & 6 & 2 & 5 & 13 \\
\hline Motorsensory & 9 & 12 & 2 & 23 \\
\hline \multicolumn{5}{|c|}{ Electrophysiological study finding } \\
\hline Axonal & 12 & 12 & 6 & 30 \\
\hline Demyelinating & 3 & 2 & 1 & 6 \\
\hline \multicolumn{5}{|c|}{ Duration of illness before biopsy } \\
\hline$<1$ year & 11 & 6 & 3 & 20 \\
\hline$>1$ year & 4 & 8 & 4 & 16 \\
\hline
\end{tabular}

Table 2 Final diagnosis in nerve biopsy group.

\begin{tabular}{|l|c|}
\hline \multicolumn{1}{|c}{ Final diagnosis } & No. of patients (\%) \\
\hline HD & $18(50 \%)$ \\
\hline Vasculitis & $8(22.32 \%)$ \\
\hline CIDP & $2(5.56 \%)$ \\
\hline Diabetic & $1(2.78 \%)$ \\
\hline Undiagnosed & $7(19.44 \%)$ \\
\hline Total & $36(100 \%)$ \\
\hline
\end{tabular}

in $78 \%$ patients.

A correlation between the clinical and electrodiagnostic findings with the nerve biopsy revealed that that impaired joint position and vibration was more in group of patients in which biopsy proved useful (Diagnostic as well as contributory), but $p$ value was not significant. Similarly diminished DTRs were statistically non-significant more in useful group. Tingling was statistically more significant in biopsy favoring group than in noncontributory group ( $p$ 0.03). Biopsy was more helpful in patients with multiple mononeuropathy group than polyneuropathy ( $p 0.003$ ). Similarly patients presenting with predominant or pure sensory complains were less likely to be diagnosed by biopsy than patients with mixed presentation ( $p$ 0.04). Pattern of electrophysiological involvement either axonal or demyelinating and duration of illness before biopsy had no correalation with the outcome of biopsy.

\section{Discussion}

This was a prospective study of 75 consecutive patients of peripheral neuropathy who referred to us and were undiagnosed after evaluation by physicians. Only thirty six of them underwent sural nerve biopsy for a disabling neuropathy. Most evident straightforward causes like Diabetes, alcohol, drug induced etc. were not included. Probable hereditary neuropathy patents were not considered for biopsy rather they went for genetic evaluation. Thus mainly the treatable cases which remained undiagnosed/ inconclusive after clinical, electro diagnostic and biochemical evaluation were considered.

In fifteen (41\%) of the patients nerve biopsies were diagnostic. In another 14 cases (39\%) patients nerve biopsy proved to be contributory. It thus helped in confirming diagnosis or excluded other causes of neuropathy thus ending the controversy. Thus in about 29 cases (80\%), performing nerve biopsy proved to be worth wile. Of least value were the seven (20\%) biopsies which showed either a non-specific axonal neuropathy or no information.

An important point to be noted here is a detailed clinical history and meticulous neurological examination with first line routine investigation, proved to be sufficient in 39 patients (52\%) who were referred to us.

In a prospective study by Gabriel et al. [1] found that the greatest diagnostic value in $14 \%$, of lesser value $70 \%$ and of least value were in $16 \%$. These results revealed that $84 \%$ patients got advantage from nerve biopsy which is closer to our results. In a retrospective study of by Hughes et al they also noted a change in diagnosis in $17 \%$ of 36 patients [7]. In one retrospective study Argov et al. [8] considered that nerve biopsy contributed to the diagnosis in $38 \%$ of 53 patients. In another, Neundorfer et al. [9] considered that the biopsy was "crucial" for establishment of the diagnosis in $27 \%$ of 56 patients and confirmed a previously suspected diagnosis in $37 \%$. In the largest retrospective study Oh [10] reported helpful or relevant information in $45 \%$ of 385 biopsies. In a study of 100 patients over the age of 65 years with disabling neuropathy, studied retrospectively, more than one third had a vasculitic neuropathy, and a further $25 \%$ had either CIDP or dysglobulinaemic neuropathy [11].

In our series leprous neuropathy was found most common (50\%) cause of undiagnosed peripheral neuropathy. This is highly different from other studies from western world. This indicates that leprosy is still most common cause of treatable peripheral neuropathy. These results also depict that leprosy is still endemic in India despite of all efforts by Government of India and WHO. Leprosy neuropathy almost always occurs in conjunction with 
a certain type of skin lesion. The presence of nerve deficit in patients from endemic areas who did not have skin lesions is considered sufficient reason for a PNL diagnosis [12-14].

In the presence of dense neuropathic sensory loss and dark skin colored people it is not always possible to make out anesthetic patch over skin so there should be a high index of suspicion.

The proportion of leprosy patients with PNL will ultimately depend on the population in question, as, for example, in India, where its incidence has been reported to range from 5.5 to $17.7 \%$ of all leprosy cases [15]. Nerve biopsy finding are well according to previous studies.

Vasculitic neuropathy was demonstrated in 8 patients, out of them in 5 patients it was associated with necrotizing arteritis and it was found very likely in 3 patients, accounting for a total of $22 \%$ of the cases. This is similar than that found in other studies [16-20]. A correlation of biopsy with the clinical features revealed that in the patients with the clinical pattern of multiple mononeuropathy, nerve biopsy proved beneficial than polyneuropathy group (P 0.003). Similarly in patients with sensorimotor symptoms nerve biopsy was more valuable ( $P$ 0.04). A significant correlation was found in presence or absence of tingling. Biopsy proved more diagnostic when tingling was there than absence of it ( $P$ 0.03). These results are expected in the case of multiple mononeuropathy.

\section{Conclusions}

Thus in carefully selected patient group nerve biopsy is a useful aid for etiological diagnosis and management of patients, more so in patients with mononeuropathy multiplex group. In this prospective planned study sural nerve biopsy altered the diagnosis in $41 \%$ and overall contributed in $80 \%$ in 36 consecutive patients. Hansen's disease was the commonest cause of neuropathy in our series and being one of the commonest treatable causes of neuropathy in an endemic country like India, one must have a high index of suspicion so that early can be started and long term complications prevented. 


\section{References}

1 Gabriel CM, Howard R, Kinsella N, Lucas S, McColl I, et al. (2000) Prospective study of the usefulness of sural nerve biopsy. J Neurol Neurosurg Psychiatry 69: 442-446.

2 Argov Z, Steiner I, Soffer D (1989) The yield of sural nerve biopsy in the evaluation of peripheral neuropathies. Acta Neurol Scand 79: 243-245.

3 Rappaport WD, Valente J, Hunter GC, Rance NE, Lick S, et al. (1993) Clinical utilization and complications of sural nerve biopsy. Am J Surg 166: $252-256$

4 Hellmann DB, Laing TJ, Petri M, Whiting-O'Keefe Q, Parry GJ (1988) Mononeuritis multiplex: the yield of evaluations for occult rheumatic diseases. Medicine (Baltimore) 67: 145-153.

5 Said G, Lacroix-Ciaudo C, Fujimura H, Blas C, Faux N (1988) The peripheral neuropathy of necrotizing arteritis: a clinicopathological study. Ann Neurol 23: 461-465.

6 Medical Research Council War Memorandum (1962) Aids to investigation of peripheral nerve injuries, ( 2 nd edn). London.

7 Hughes RAC, Adams CWM, Hall S, et al. (1990) The contribution of nerve biopsy to the management of peripheral nerve disease. $J$ Neurol Neurosurg Psychiatry 53: 714.

8 Argov Z, Steiner I, Soffer D (1989) The yield of sural nerve biopsy in the evaluation of peripheral neuropathies. Acta Neurol Scand 79: 243-245.

9 Neundörfer B, Grahmann F, Engelhardt A, Harte U (1990) Postoperative effects and value of sural nerve biopsies: a retrospective study. Eur Neurol 30: 350-352.
10 Oh SJ (1990) Diagnostic usefulness and limitations of the sural nerve biopsy. Yonsei Med J 31: 1-26.

11 Chia L, Fernandez A, Lacroix C, Adams D, Planté V, et al. (1996) Contribution of nerve biopsy findings to the diagnosis of disabling neuropathy in the elderly. A retrospective review of 100 consecutive patients. Brain 119: 1091-1098.

12 Kaur G, Girdhar BK, Girdhar A, Malaviya GN, Mukherjee A, et al. (1991) A clinical, immunological, and histological study of neuritic leprosy patients. Int J Lepr Other Mycobact Dis 59: 385-391.

13 Mahajan PM, Jogaikar DG, Mehta JM (1996) A study of pure neuritic leprosy: clinical experience. Indian J Lepr 68: 137-141.

14 Uplekar MW, Antia NH (1986) Clinical and histopathological observations on pure neuritic leprosy. Indian J Lepr 58: 513-521.

15 Talwar S Jha PK, Tiwari VD (1992) Neuritic leprosy: epidemiology and therapeutic responsiveness. Lepr Rev 63: 263-268.

16 Hessel L, Corvisier N, Vallat JM, Michel JP (1986) Etiologies des neuropathies peripheriques chez les personnes agees. Medecine Hygiene 44: 1354-1360.

17 Huang CY (1981) Peripheral neuropathy in the elderly: a clinical and electrophysiologic study. J Am Geriatr Soc 29: 49-54.

18 George J, Twomey JA (1986) Causes of polyneuropathy in the elderly. Age Ageing 15: 247-249.

19 Barach JK (1989) Peripheral neuropathy (PN) in elderly population (65 years and above). Neurology 39: 208.

20 Wertman E, Zilber N, Abramsky O (1988) Clinical features of peripheral neuropathy in the elderly. J Neurol 235: S44 